\title{
Relationships of Nerve Conduction Parameters with the Thyroid Hormones in Hypothyroid Patients
}

\author{
Sabina Yeasmin ${ }^{1}$, Noorzahan Begum ${ }^{2}$, Shelina Begum ${ }^{3}$, Shah M Hafizur Rahman ${ }^{4}$
}

\begin{abstract}
Background: Impairment of nerve conduction may occur in hypothyroidism which usually develops insidiously over a long period of time due to irregular taking of drugs or lack of thyroid hormone replacement. Objectives: To evaluate the thyroid hormone status and the clinical and electrophysiological changes in hypothyroid patients in order to observe their relationships with nerve conduction changes. Methods: Thirty healthy euthyroid subjects with the age range from 20 to 50 years of both sexes were as control and 15 hypothyroids with TSH with $<60 \mathrm{MIU} / \mathrm{L}$ and the duration of 6 months to 5 years were as experimental. Serum $\mathrm{TT}_{3}, \mathrm{TT}_{4}$ were measured by RIA and IRMA method. The distal latency (D L) and Nerve Conduction Velocities (NCV) for sensory and motor function were measured by a standard electrophysiological technique in median and ulnar nerve for upper limb and for lower limb, in sural nerve for sensory function and common peroneal nerve for motor function. Data were analyzed statistically by unpaired ' $t$ ' test, $\mathrm{Z}$ test, Pearson's correlation coefficient test. Results: Both $\mathrm{TT}_{3}, \mathrm{TT}_{4}$ levels were significantly $(\mathrm{P}<0.001)$ lower in hypothyroids. Again, $60 \%$ hypothyroids and $10 \%$ euthyroids $(\mathrm{P}<.001)$ had abnormal NCV. The relationships of TSH with both the median and ulnar sensory and motor distal latencies, common peroneal motor and sural sensory distal latencies and ulnar sensory conduction velocity were positive. But these relationships were negative with the median and common peroneal motor nerve conduction velocities, sural sensory conduction velocities. Only the relationships between median sensory distal latency and TSH was statistically significant $(\mathrm{P}<0.05)$. Again, a positive correlation of $\mathrm{TT}_{3}$ and $\mathrm{TT}_{4}$ with the median, and sural sensory and common peroneal motor conduction velocities, but negative correlation with median motor and sural sensory latencies were found. In addition, positive correlation between $\mathrm{TT}_{3}$ and ulnar sensory and between $\mathrm{TT}_{4}$ and Ulnar motor conduction velocities were observed. Again the relationships median and ulnar sensory latencies were negative with $\mathrm{TT}_{4}$ only. The value of coefficient between $\mathrm{TT}_{4}$ and sural distal latency and conduction velocities were statistically significant $(\mathrm{P}<0.05)$. Conclusion: Results of this study showed that the impairment of nerve conduction occurs in hypothyroidism.
\end{abstract}

Key Words: Hypothyroidism, Pearson's correlation, nerve conduction.

J Bangladesh Soc Physiol. 2013 June; 8(1): 1-5 For Authors Affiliation, see end of text.

http://www.banglajol.info/index.php/JBSP

\section{Introduction}

H ypothyroidism may lead to nerve conduction impairment which develops insidiously over a long time due to

Received; June 2012 Accepted : September 2012

J Bangladesh Soc Physiol. 2013, June; 8(1): 1-5 irregular taking of drugs or lack of hormone replacement therapy and the severity and duration of thyroid deficiency may affect the of clinical pictures in hypothyroidism. ${ }^{1}$ The investigators studied nerve conduction parameters like distal 
latencies and nerve conduction velocities in hypothyroid patients to observe the nerve conduction impairment and functional status of peripheral nerves in thyroid deficiency. Investigators observed the increased in distal latency with decreased in conduction velocities in the peripheral nerves with the patients of hypothyroidism that might be an indication of nerve conduction impairment. $^{2}$

The investigators studied nerve conduction parameters in hypothyroid patients to observe the functional status of peripheral nerves in thyroid deficiency. ${ }^{2-4}$ Most of them had shown that deficiency of thyroid hormones cause nerve conduction impairment of different peripheral nerves but more commonly the median nerve. The common nerve conduction parameters done by the investigators include sensory motor distal latencies (dl), sensory motor conduction velocities (NCV) in different peripheral nerves. Conduction impairment of the nerve revealed by the increased distal latency ( $\mathrm{dl}$ ) and decreased conduction velocities (NCV) in that nerve and investigator s also suggested that the nerve conduction impairment is frequent in the advanced hypothyroidism. ${ }^{2-4}$

So, it can be said that nerve dysfunctions are common in thyroid deficiency and there is a relationship of the nerve conduction impairment with the hormonal status in hypothyroidism. These findings are in agreement with the findings of some other investigators ${ }^{3}$.

Some of the previous study had mentioned that the severity and duration of hypothyroidism may have some relations with the clinical pictures of nerve conduction impairment but no study has yet been carried out on that aspect in our country. ${ }^{2-4}$ Moreover, a few data regarding the normal values of nerve conduction parameters of healthy Bangladeshi population are available 5 but no data in hypothyroid patients on these aspects are yet available.

So, this study has designed to find out the relationships of nerve conduction parameters with the thyroid status in hypothyroid patients with an aim to give a guideline to the physicians for proper and better management of the patients and also to create awareness among these group of patients so that they can take early treatment and prevent the complications like nerve conduction impairment.

\section{Methods}

A total number of 45 subjects with the age range of 20 to 50 years of both sexes were included in this study, of whom 30 euthyroids $(\mathrm{TSH}=0.3$ $5 \mathrm{MIU} / \mathrm{L}$ ) were included in group A (control) and 15 hypothyroids ( $\mathrm{TSH}<60 \mathrm{MIU} / \mathrm{L}$ ) were included in group B (study group). The duration of the disease varied from 6 months to 5 years. The objectives of the study were explained to each of the subjects and their written consents were taken. Detailed medical history was taken regarding drug intake and general and clinical examinations were done. The hormones were measured by $\mathrm{RIA}^{6,7}$ for $\mathrm{TT}_{3}$ and $\mathrm{TT}_{4}$ and $\mathrm{IRMA}^{8}$ for TSH. The nerve conduction studies were done by electrophysiological method with a standard NCV machine ${ }^{3,4}$.

The statistical analysis was done by unpaired $t$ test, Z and Pearson's correlation coefficient test.

\section{Results}

All the parametric variables were expressed as mean $( \pm \mathrm{SD})$. The comparison of the values was done among the different groups. In this study, the mean $\mathrm{TT}_{3}$ and $\mathrm{TT}_{4}$ were significantly lower in hypothyroids in comparison to those of healthy (Table I). Again, nerve conduction study also revealed, 9 (60\%) of the hypothyroid subjects had the nerve conduction impairment, where as only $3(10 \%)$ of euthyroid showed this, but $27(90 \%)$ of them had normal study (Table II).

Table I : Serum $\mathrm{TT}_{3}$ and $\mathrm{TT}_{4}$ and TSH levels of the study subjects $(n=45)$

\begin{tabular}{lccc}
\hline Parameters & $\begin{array}{c}\text { Group A } \\
(\mathrm{n}=30)\end{array}$ & $\begin{array}{c}\text { Group B } \\
(\mathrm{n}=15)\end{array}$ & $\begin{array}{c}\text { P } \\
\text { values }\end{array}$ \\
\hline $\mathrm{TT}_{3}(\mathrm{nmol} / \mathrm{L})$ & $2.18 \pm 0.53$ & $1.31 \pm 0.81$ & $<0.001$ \\
$\mathrm{TT}_{4}(\mathrm{nmol} / \mathrm{L})$ & $129 \pm 28.51$ & $61.21 \pm 29.81$ & $<0.001$ \\
$\mathrm{TSH}(\mathrm{MIU} / \mathrm{L})$ & $0.76-4.8$ & $7-<60$ & - \\
\hline
\end{tabular}

Results are expressed as Mean $\pm \mathrm{SD}$. Unpaired ' $\mathrm{t}$ ' test has been done as the test of significance, Group $A=$ Euthyroids (Control group), Group $\mathrm{B}=$ Hypothyroids with TSH level $<60 \mathrm{MIU} / \mathrm{L}$

J Bangladesh Soc Physiol. 2013, June; 8(1): 1-5 
Table II : Distribution of subjects by NCV $(n=45)$

\begin{tabular}{lcc}
\hline Parameters & Group A & Group B \\
& No $(\%)$ & No $(\%)$ \\
\hline Normal NCV & $27(90)$ & $6(40)$ \\
Abnormal NCV & $3(10)$ & $9(60) * * *$ \\
\hline
\end{tabular}

Statistical analysis was done by ' $Z$ ' test, $* * *=\mathrm{P}<0.001$, Group $\mathrm{A}=$ Euthyroid (control) group, Group $\mathrm{B}=\mathrm{Hyp}$ thyroid group, $\mathrm{NCV}=\mathrm{Nerve}$ conduction velocity, $n=$ Total no of subjects

The relationships of the nerve conduction parameters with the TSH level (Table III) revealed positive relationships with the median and ulnar sensory and motor distal latencies, ulnar sensory conduction velocity, common peroneal motor distal latency and sural sensory distal latency, But the relationships were negative with the median and common peroneal motor nerve conduction velocities, sural sensory conduction velocities. Among these, the relationship of TSH and median sensory distal latency was only statistically significant $(\mathrm{P}<0.05)$.

Relationships of the nerve conduction parameters with $\mathrm{TT}_{3}$ showed a positive relationships with the sensory conduction velocities $(\mathrm{NCV})$ of median, ulnar and sural nerves, the sensory distal latencies (dl) of median and ulnar nerves, motor conduction velocities $(\mathrm{NCV})$ of common peroneal nerve, motor latencies (dl) of ulnar and common peroneal nerves where as the negative relationships were observed with median motor distal latency (dl) and conduction velocities (NCV), ulnar motor conduction velocity and sural sensory latency (dl), although none of all these relationships were statistically significant.

Relationships between $\mathrm{TT}_{4}$ and nerve conduction parameters showed a positive relationship with the Median and sural sensory and ulnar and common peroneal motor conduction velocities (NCV), ulnar and common peroneal motor latencies (dl) but the relationships were negative with the median, ulnar and sural sensory distal latencies (dl) and median motor and ulnar sensory conduction velocities(NCV). Among these, the relationships of $\mathrm{TT}_{4}$ with sural distal latency $(\mathrm{dl})(\mathrm{P}<0.05)$ and sural nerve conduction velocities $(\mathrm{NCV})(\mathrm{P}<0.05)$ were only statistically significant.

Table III: Correlation of nerve conduction parameters with $\mathrm{TT}_{3}, \mathrm{TT}_{4}$ and $\mathrm{TSH}$ level in hypothyroid subjects $(\mathrm{n}=15)$

\begin{tabular}{lccc}
\hline Variables & $\mathrm{TSH}$ & $\mathrm{TT}_{3}$ & $\mathrm{TT}_{4}$ \\
& $\mathrm{r} / \mathrm{P}$ & $\mathrm{r} / \mathrm{P}$ & $\mathrm{r} / \mathrm{P}$ \\
\hline M d latency(s) & $+0.541 / 0.03^{*}$ & $+0.274 / 0.322$ & $-0.275 / 0.321$ \\
M NCV $(\mathrm{s})$ & $-0.422 / 0.117$ & $+0.246 / 0.376$ & $+0.291 / 0.293$ \\
M d latency (m) & $+0.037 / 0.897$ & $-0.70 / 0.804$ & $-0.451 / 0.092$ \\
MNCV (m) & $-0.232 / 0.406$ & $-0.352 / 0.198$ & $-0.215 / 0.443$ \\
U d latency (s) & $+0.073 / 0.796$ & $+0.185 / 0.510$ & $-0.352 / 0.199$ \\
U NCV (s) & $+0.012 / 0.966$ & $+0.172 / 0.539$ & $-0.038 / 0.893$ \\
Ud latency (m) & $+0.230 / 0.411$ & $+0.314 / 0.225$ & $+0.070 / 0.804$ \\
UNCV (m) & $-0.240 / 0.389$ & $-0.025 / 0.929$ & $+0.320 / 0.245$ \\
CP d latency (m) & $+0.222 / 0.426$ & $+0.348 / 0.204$ & $+0.095 / 0.737$ \\
CPNCV (m) & $-0.237 / 0.394$ & $+0.063 / 0.823$ & $+0.368 / 0.177$ \\
S d latency (s) & $+0.280 / 0.312$ & $-0.155 / 0.582$ & $-0.574 / 0.025^{*}$ \\
S NCV (s) & $-0.331 / 0.228$ & $+0.047 / 0.869$ & $+0.535 / 0.040^{*}$ \\
\hline
\end{tabular}

$\mathrm{Md}$ latency $=$ Median distal latency, $\mathrm{M} \mathrm{NCV}=$ Median nerve conduction velocity, $\mathrm{U} d$ latency $=$ Ulnar distal latency, $\mathrm{U} \mathrm{NCV}=$ Ulnar nerve nerve conduction velocity, CP D latency $=$ Common peroneal distal latency, $\mathrm{CP} \mathrm{NCV}=$ Common peroneal nerve conduction velocity, $\mathrm{S} d$ latency $=$ Sural distal latency, $\mathrm{S}$ NCV $=$ Sural nerve conduction velocity, $\mathrm{s}=$ sensory, $\mathrm{m}=$ motor, $(-)=$ Negative correlation, $(+)=$ Positive correlation $*=\mathrm{P}<0.05 . \mathrm{n}=$ Total no of subjects 


\section{Discussions}

In this study, all the hypothyroid patients had significantly lower $\mathrm{TT}_{3}$ and $\mathrm{TT}_{4}$ levels compared to euthyroids.

The relationships of TSH with median distal latency (sensory) showed a statistically significant positive relationship only, The relationships of TSH with most of the nerve conduction parameters showed a relevant correlations but they were not statistically significant. These findings may be explained by the fact that the thyroid deficiency may be a factor for the deterioration of nerve conduction parameters. ${ }^{7}$

Relationships of all the nerve conduction parameters with serum $\mathrm{TT}_{3}$ were statistically non significant. Inability to find the significant correlations between nerve conduction parameters and $\mathrm{TT}_{3}$ level in this study may be due to their almost normal level of $\mathrm{TT}_{3}$ as most of the hypothyroid subjects were on hormone therapy.

Relationships of $\mathrm{TT}_{4}$ showed a statistically significant negative correlations with Sural distal latency and positive correlations with Sural NCV. This findings also correlate the deterioration of nerve conduction with the decreased hormone. Except these, the positive relationships with ulnar and common peroneal motor latencies, median sensory, ulnar and common peroneal motor conduction velocities and the negative relationships with median motor latency and motor conduction velocity and ulnar sensory latency and sensory conduction velocity were statistically non significant.

The mechanisms of deterioration of nerve conduction parameters in hypothyroidism are not yet fully clear but different investigators suggested that the weight gain in hypothyroids may be a contributory factor for this nerve conduction abnormalities. In addition, the deposition of mucopolysaccharide or the myxedematous tissue may also results in compression over the peripheral nerves and thereby swelling and degeneration of them. ${ }^{2,4}$

It has also been suggested that the thyroid hormones stimulate the mitochondrial respiratory activity to produce ATP during aerobiosis under normal physiological condition. Hormones also increase the ATPase activity and consequently $\mathrm{Na}^{+} / \mathrm{K}^{+}$pump activity in this group of patients. Therefore, deficiency of ATP and reduced ATPase and decreased $\mathrm{Na}^{+} / \mathrm{K}^{+}$pump activity may be responsible for this changes in this group of patients. ${ }^{1}$ Decrease glycogen degradation may also leads to energy deficit in hypothyroid patients. ${ }^{1,3,4,7}$ In this study, both these factors may be responsible for nerve conduction impairment in hypothyroids.

The non significant correlations between the thyroid hormones and most of the nerve conduction parameters of the present study may be due to the almost normal values of $\mathrm{TT}_{3}$ and $\mathrm{TT}_{4}$ in hypothyroid subjects as most of them were on hormone therapy.

\section{Conclusion}

So all the findings of the study revealed that the impairment of nerve conduction parameters may occur in hypothyroids. ${ }^{9}$

\section{Authors Affiliations}

* 1. Sabina Yeasmin, Associate Professor of Physiology. Dr Sirajul Islam Medical College, Mogbazar, Dhaka, Bangladesh.

2. Noorzahan Begum, Professor and Ex Chairman, Department of Physiology, Bangabandhu Sheikh Mujib Medical University (BSMMU), Shahbag, Bangladesh

3. Shelina Begum, Professor and Chairman, Department of Physiology, Bangabandhu Sheikh Mujib Medical University (BSMMU), Shahbag, Bangladesh.

4. Shah Mohammed Hafizur Rahman, Assistant Professor of Orthosurgery, Center for Medical Education (CME), Mohakhali, Dhaka.

\section{References:}

1. Nemni R, Bottacchi E, Fazio R Et al. Poly neuropathy in hypothyroidism: Clinical, Electrophysiological and morphological findings in 4 cases. J Neurol Neurosurg Psychiatry 1987; 50: 1454-1460.

J Bangladesh Soc Physiol. 2013, June; 8(1): 1-5 
2. Preston D C. Index normal adult values In: Electromyography and Neuromuscular Disorders, Clinical - Electrophysiological Correlations, Butterworth- Heinemann. USA 1998; pp 561-565.

3. Rao SN, Katiyar BC, Nair KRP, Misra S. Neuromuscular status in hypothyroidism. Acta Neurol Scand 1980; 61:167-177.

4. Shirabbe T, Tawara S, Tetrao A, Araki S. Myxedematous polyneuropathy: a light and electron microscopy study of peripheral nerve and muscle. J Neurol Neurosurg Psychiatry 1975; 38: 241-247.

5. Sultana S. Some aspects of electrophysiological changes of peripheral nerves in diabetic patients of different duration [M Phill thesis] Dhaka:
Bangabandhu Sheikh Mujib Medical University 2003.

6. Total Triiodothyronine $\left(\mathrm{T}_{3}\right)$ Radioimmunoassay Kit (PR) IMK-422 [Manual] 2005, Department Of Isotope. China Institute of Atomic Energy, Beijing.

7. Total Thyroxine $\left(\mathrm{T}_{4}\right)$ Radioimmunoassay Kit $(\mathrm{PR})$ IMK-419 [Manual] 2005, Beijing Atom Hightech Co., Ltd., Beijing.

8. TSH Immunoradiometric assay Kit IMK-432 [Manual] 2005, Beijing Atom Hightech Co., Ltd., Beijing.

9. Torres CF, Moxley RT. Hypothyroid neuropathy and myopathy: clinical and electrodiagnostic longitudinal findings. J Neurol 1990; 237: 271274. 